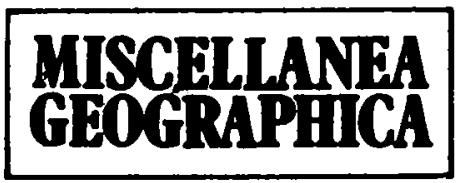

W A S Z A W A 1988

Roman Domachowski

\title{
L'ENSEIGNEMENT DE LA GEOGRAPHIE A L'ECOLE PRIMAIRE POLONAISE
}

La notion de „programme de l'enseignement” a, en Pologne, un sens multiple - il existe autant de définitions que d'auteurs. Selon Wincenty Okoń, „le programme est' une présentation d'objectifs, de contenu et de méthodes d'enseigner et d'apprendre une matière scolaire donnée, parfois aussi des résultats à obtenir des élèves" (Okoń, 1984). En suivant la pensée de Heliodor Muszyński, Anna Bogdańska souligne le caractère téléologique du programme - la fonction du programme consiste à décrire ,non pas ce que l'on doit faire à l'école, mais ce qu'on doit y obtenir (Bogdańska, 1978). Il est plutôt facile de préciser la portée des objectifs à atteindre, mais les méthodes de leur réalisation peuvent être nombreuses. Ainsi, dans' la communication présente, le programme est considéré comme un système d'objectifs et de contenus indispensables que l'élève doit atteindre grâce à la réalisation dudit programme.

L'explication de la structure du programme nécessite la connaissance non seulement de ses éléments et des relations entre ces derniers, mais également celle du système didactique dans lequel ledit programme devrait fonctionner. En dehors du programme de l'enseignement, dans le système didactique entrent également les conditions nécessaires à la réalisation de ce programme. Conformément aux principes du système didactique en vigueur dans notre pays, le programme de l'ensèignement de chaque matière doit permettre d'équilibrer l'enseignement et l'éducation, les deux étant considérés comme deux pôles du même processus. Le principe de l'intégration de l'enseignement et de l'éducation, obligatoire dans la didactique polonaise, admet la nécessité d'influer sur toutes les sphères de la personnalité de l'élève. Partant de ce principe, le programme de l'enseignement de la géographie doit tenir compte de trois objectifs, à savoir: les connaissances, le savoir, et les valeures idéologico-morales.

On a pris en considération des principes du domaine de la psychologie de développement. Le programme a été établi de façon à ce que tous les efforts à accomplir par les élèves afin de réaliser ledit programme soient conformes au niveau d'âge de ses destinaires. 
Le choix de matières contenues dans le programme de l'enseignement de la géographie résulte du développement de la géographie elle-même. Compte tenu d'une grande diversité des sciences géographiques en ce qui concerne l'objet et les méthodes de recherche, ainsi que du processus de spécialisation qui ne cesse de progresser, le programme a été enrichi de matières permețtant de comprendre le caractère de la géographie en tant qu'un ensemble de disciplines scientifiques défini comme la ,géographie". En tant que science, la géographie se compose de nombreuses notions et thèses, et se caractérise par ses propres méthodes de parvenir aux généralisations. Certaines de ses notions caractérisent la géographie dans son ensemble, en définissant son type logique, d'autres se rapportent à ses branches particulières, allant jusqu'aux moins générales, concernant les résultats détaillés de recherches (Domański, 1977). Ceci a une grande importance pour l'économie de l'enseignement, car si l'on admet l'existence d'ensembles de notions représentatives pour une discipline donnée, leur compréhension entraînerait une compréhension des éléments détaillés des connaissances composant cette discipline. Voilà l'un des principes de base de l'établissement de nouveaux programmes de l'enseignement de la geographie. Les matières ont été choisies de façon permettant à l'élève de comprendre les lois principales de la géographie (p.ex., la loi de la zonalité aussi bien horizontale que verticale, les lois de la dialectique des processus naturels, le principe de l'actualisme, etc.) et de connaître les territoires de la Terre différenciés du point de vue des caractéristiques naturelles et anthropogéniques.

Le choix de matières enseignées destinées aux différents niveaux de l'enseignement (classes) était fait conformément aux trois critères, à savoir: conformité aux principa $x$ objectifs de l'enseignement, conformité aux phases successives du déve ioppemer i psychique des enfants et des adolescents, utilisation des acquis der sciences séographiques.

Conformément aux shases c $d$ développement psycho-physique des jeunes, les programmes destinés à r'école primaire* ont deux degrés suivants: niveau primaire (de la première à la troisième classes) et niveau moyen (de la quatrième à la huitième classes). Au premier niveau, la géographie n'est pas encore une matière indépendante, mais la matière portant le nom d',Environnement socio-naturel" apporte une grande quantité de notions géographiques. Le degré moyen comporte deux niveaux, à savoir: systématisation préliminaire (la quatrième et la cinquième classes) et enseignement systématique (de la sixième à la huitième classes). Les programmes de la géographie destinés à chaque niveau sont différents du point de vue de l'étendue du matériel à apprendre, la structure de celui-ci et la façon de l'interpréter. Le choix du matériel au niveau de la systématisation préliminaire est particulièrement difficile, car la compré-

* Les classes en Pologne sont numérotées à la façon inverse de la France, et commencent par la première. 
hension de certaines notions introduites à ce niveau (p.ex. état de l'atmosphère, climat, sol, etc.) exige d'un élève de faire abstraction des objets concrets et, partant de l'analyse et de la synthèse, de saisir les traits formels caractérisant non pas un seul objet (phénomène) mais tout un groupe de phénomènes. Ainsi, l'enseignement de la géographie générale (physique et économique) commence au niveau de l'enseignement systématique (de la sixième à la huitième classes). Les interdépendances très complexes existant entre l'environnement géographique et l'activité de l'homme ne peuvent pas être comprises par les moins de quatorze ans. Donc, les problèmes relatifs aux règles de la localisation d'activités économiques ne sont introduites qu'au programme de la huitième classe, et sont présentés sur les exemples polonais. Au niveau de la systématisation générale, le matériel apportant des notions et familiarisant l'élève avec l'idée de l'abstraction est enrichi de matériel factographique et de matériel d'observation, les deux permettant d'atteindre les généralisations par voie de la réunion inductive de données.

$\mathrm{Au}$ premier niveau de l'enseignement, le programme a le caractère épisodique, c'est-à-dire ne présente que des fragments choisis de la réalité, sans faire un cours systématique. Le programme de la géographie destiné à la quatrième et à la cinquième classes a été établi partant de la conception de la description exemplaire du paysage. Le but de ce programme consiste à familiariser les élèves avec certains problèmes généraux relevant de la géographie physique et de la géographie économique dont les éléments seront introduits aux programmes respectifs des classes suivantes. Ce but sera réalisé au moyen de la présentation des paysages choisis permettant de procéder aux généralisations des caractéristiques choisies desdits paysages. Les programmes respectifs de la quatrième et de la cinquième classes ont été conçus de manière à familiariser les étèves avec certains types de paysages, sur l'exemple d'unités régionales déterminées. Néanmois, ce n'est pas encore un cours de la géographie régionale. Dans les descriptions régionales, on a recours, en premier liéu, à la méthode déductive, au contraire des descriptions exemplaires où l'on applique la méthode inductive (Flis, 1983). Dans le programme de la quatrième classe, le choix des paysages décrits a été fait en fonction du type du relief, on a également tenu compte des types de paysages mis en évidence eu égard à la façon de l'aménagement économique du terrain. En cinquième classe, l'élève fait connaissance des types de paysages du mode entier. Leur choix a eté fait conformément aux critères de la zonalité, de l'étagement et des-influences marines; on a également inclus à ce programme les paysages classifiés selon le critère géologique et selon le type du relief. En dehors des paysages choisis conformément au critère naturel, on présente également les paysages anthropogéniques. La revue de paysages choisis a été précédee par un cours:

- des connaissances générales de la Terre;

- des rudiments de l'orientation sur la Terre, compte tenu du réseau géographique, 


\section{- de la projection cartographique de la Terre.}

Les connaissances mentionnés plus haut sont indispensables à l'enseignement de la deuxième partie du programme. Le cours de la géographie en cinquième classe se termine par la présentation des mouvements de la Terre et de certaines de leurs conséquences, ensuite on passe à la distinction, sur les exemples déjà connus, des types de paysages zonaux et azonaux, et à celle des types de paysages transformés par l'homme. L'assimilation de ce programme permet la réalisation d'un cours de la géographie régionale prévu pour les classes suivantes.

Les programmes de la sixième et de la septième classes sont plus complexes. La conception de l'enseignement de la géographie régionale tient compte de l'approche systémique dans les recherches poursuivies dans cette discipline, conformément à laquelle la région est considérée comme un système. Cette approche perrmet de connaître les lois régissant chaque élément de l'environnement, ainsi que les principes de fonctionnement du système, autrement dit de la région. La partie finale du programme de la septième classe est une sorte de synthèse de l'enseignement régional de la géographie. Cette partie est intitulée „Problèmes socio-économiques du monde contemporain". Le but principal de cette partie du programme consiste à trouver la réponse aux questions suivantes: comment l'homme utilise les valeurs différenciées de l'environnement? quel est l'état de celui-ci étant donné l'activité humaine? enfin, quelles sont les perspectives du développement ultérieur de la civilisation? La présentation des processus et des formes d'intégration, et d'une coopération internationale constitue une synthèse effectuée à partir des problèmes socio-économiques.

Un cours de géogra hie de la Pologne en huitième classe clôt l'enseignement de la gé graphie lans l'école primaire. Le programme consiste en plusieurs $\mathrm{p}$ ties. Ai si, l'introduction se réfère au passé historique de notre pays; la partıe suivante caractérise l'environnement géographique de la Pologne, la dernière partie, ènfỉn, caractérise ses régions géographiques. A l'occasion de la présentation des régions physico-géographiques, on peut expliquer plusieurs théories: ainsi, p.ex., les élèves font connaissance de la genèse de certaines roches et de certaines structures géologiques, et de la genèse du relief glacial; ensuite on explique l'étagement du paysage de la montagne, et la formation des précipitations orographiques; enfin, on presente certains des principes de la localisation des activités économiques. Partant de la caractéristique régionale, on aborde les problèmes propres à l'ensemble du territoire polonais. Le cours de la géographie destiné à l'école primaire se termine par la partie intitulée „Région où je vis". Après s'être familiarisés avec la différenciation régionale et avec les problèmes-clés de notre économiè, les élèves se penchent sur les problèmes de la région où ils habitent.

La réalisation du programme de la géographie à l'école primaire devrait fournir aux élèves les rudiments des connaissances dé la différenciation de 
l'environnement géographique et de l'activité économique de l'homme à l'échelle locale, régionale et globale. En dehors des connaissances théoriques, les élèves doivent assimiler certaines connaissances pratiques.

Les connaissances pratiques propres à la géographie peuvent être réparties en quatre groupes:

a) des travaux auxiliaires de type technique dont la lecture de différentes cartes, l'exécution des croquis topographiques ou l'utilisation de données statistiques;

b) la localisation de phénomènes dans l'espace géographique;

c) l'observation d'objets et de phénomènes géographiques, leur classification et la perception des relations de cause à effet;

d) l'estimation critique de l'activité humaine dans l'environnement géographique.

Les quatre groupes de connaissances pratiques cités plus haut sont réalisés au cours du cycle d'enseignement au niveau primaire. Le programme destiné à chaque classe prévoit leur réalisation sous forme plus détaillée. Voici comment seront réalisés différents groupes de connaissances pratiques conformément au niveau de l'enseignement:

\section{De la quatrième à la cinquième classes:}

a) apprendre à se servir d'instruments tels que thermomètre, compas, boussole, géodimetre et niveau; évaluer les distances et les hauteurs relatives; déterminer les directions principales et indirectes; lire la carte hypsométrique; désigner le méridien sur le terrain; définir les directions sur le terrain, le globe terrestre et sur la carte; lire les cartes hypsométriques et de paysage;

b) apprendre à situer des objets géographiques connus (régions, villes, fleuves, configurations) par rapport aux points cardinaux et par rapport les uns aux autres; établir une situation géographique sur une carte au moyen des coordonnées géographiques;

c) apprendre à déterminer différents états de l'atmosphère - distinguer les éléments du paysage naturel des éléments du paysage transformé par l'homme; distinguer des formes principales du relief; rapprocher les symboles sur la carte et les caractéristiques du paysage; mener des observations systématiques de la sphère céleste; comparer différents paysages;

d) apprendre à évaluer les transformations du paysage dues à l'industrie; comprendre la nécessité de protéger le paysage (sur l'exemple de la Région industrielle de Haute-Silésie, de la Région de Grands Lacs de Mazurie); De la sixième à la septième classes

a) lecture des cartes topographiques et chorographiques générales et thématiques; interprétation de données statistiques et de diagrammes statistiques; orientation correcte dans le terrain à l'aide d'une carte topographique (éventuellement touristique); utilisation de la littérature et d'autres sources de l'information géographique;

b) détermination de la situation des continents et d'autres objets du globe; détermination de la situation géographique des régions et des pays connus; 
c) perception de la relation entre l'éclairement de la Terre et la circulation atmosphérique, entre les mouvements de ia Terre et les directions des vents, entre la répartition des précipitations atmosphériques et l'éclairement de la Terre; perception et explication d'une interdépendance entre les éléments naturels du paysage, les rapports démographiques et l'économie;

d) estimation de l'environnement géographique du point de vue de l'activité de l'homme; connaissance de notions de l'utilisation de ressources naturelles (matières premières, ressources en eau):

\section{La, huitième classe}

a) lecture et interprétation des cartes thématiques ; utilisation de sources d'information statistique et interprétation de données; utilisation d'autres sources d'information; présentation de phénomènes géographiques à l'aide des méthodes géographiques;

b) détermination de la situation des centres de pression formant l'état de l'atmosphère en Pologne; détermination de la situation géographique d'objets géographiques en Pologne;

c) observation des phénomènes naturels, économiques et sociaux; apprendre à tirer les conclusions à partir des données ainsi réunies;

d) évaluation de l'activité économique de l'homme dans l'environnement. L'analyse du programme démontre qu'aux niveaux les moins élevés de l'enseignement correspondent les connaissances pratiques des catégories moins élevées. Les objectifs les plus complexes liés avec la connaissance des relations' de cause à effet n'apparaissent qu'aux niveaux plus élevés de l'enseignement en rapport avec le niveau de la maturité de l'esprit des élèves.

\section{BIBLIOGRAPHIE}

O k oń, W., 1984, Slounik pedagogiczny (Dictionnaire pédagogique), PWN, Warszawa.

B og d a ń s a, A., 1978, Zasady konstruowania norm wymagań (Principes d'établissement des normes des exigences), ronéo, Warszawa.

D o ma ń sk i, R., Geografia ekonomiczna, 1977, Warszawa-Poznań.

Flis, J., 1983, „Opis krajobrazu” (Description du paysage), Geografia w szkole, n` 1. 